\title{
Prosthodontic Rehabilitation of Cleft Lip and Palate Patients using Conventional Methods: A Case Series
}

\author{
Ozlem Acar, Burcak Kaya, Muhammet Saka, Bulem Yuzugullu
}

\begin{abstract}
This case series describes prosthodontic management of seven cleft-lip-and-palate patients with different cleft deformities, gender and age. Patients were rehabilitated using conventional prosthesis. Five patients in whose maxillary arch was orthodontically expanded were treated with a combination of fixed and removable partial dentures with precision attachments. Two patients, who did not maxillary collapse were treated with metal-ceramic fixed-partial-dentures. All patients were followed up to two years. When there are limitations for secondary bonegrafting and implants, conventional approaches provide good physiologic, functional and esthetic outcomes with care taken to prevent maxillary relapse in treating these patients.
\end{abstract}

Keywords: Cleft lip and palate, Deformities, Fixed and removable prosthodontics, Orthodontics.

How to cite this article: Acar O, Kaya B, Saka M, Yuzugullu B. Prosthodontic Rehabilitation of Cleft Lip and Palate Patients using Conventional Methods: A Case Series. Int J Prosthodont Restor Dent 2013;3(3):120-124.

Source of support: Nil

Conflict of interest: None

\section{INTRODUCTION}

Clefts of the lip and palate (CLP) are commonly encountered congenital anomalies, affecting one in seven hundred live births ${ }^{1}$ and often result in severe functional deficiency on the patient's chewing abilities, appearance and ability to speak. ${ }^{2}$ Generally, they are classified into four major types: cleft lip, cleft palate, unilateral cleft lip and cleft palate (UCLP), and finally bilateral cleft lip and palate (BCLP). A combination of cleft lip and palate is the most common clefting deformity seen. The prevalence of dental anomalies associated with cleft lip and palate is remarkable. ${ }^{2}$ Abnormalities of tooth number, size, morphology, calcification and eruption have been described. ${ }^{2}$ Paranaiba et $\mathrm{al}^{3}$ demonstrated that patients with unilateral CLP were frequently more affected by dental anomalies than bilateral CLP. The incidence of congenitally missing teeth, especially lateral incisors adjacent to the alveolar cleft is high., ${ }^{2,4}$ If not missing, these teeth may be malformed and malposed. ${ }^{4}$

It is clear that multidisciplinary treatment planning by surgeons, orthodontists and restorative dentists is required for the long-term benefit of this small but challenging group of patients. ${ }^{5}$ Today, knowledge of craniofacial growth has increased, there with the success of surgical and orthodontic treatment has improved. This requires less prosthetic intervention. Nonetheless, if the edentulous cleft side is not closed ortodontically or surgically some types of prosthetic treatment is required and still, the prosthodontist remains an integral member of the cleft and craniofacial rehabilitation team. ${ }^{4}$

Maxillofacial prosthetic treatments offer improvement in function, appearance, and health of patients with congenital and craniofacial defects. When planning a prosthetic rehabilitation for a patient with congenital abnormalities; lack of teeth, intraoral anatomic deformities, inadequate arc development, and inspection of appropriate occlusal vertical dimension must be taken into consideration. ${ }^{6}$ There are various treatment modalities of definitive prosthesis for unilateral and bilateral cleft lip and palate patients after completion of orthodontic treatment such as; conventional multi-unit fixed partial denture (FPD), resin composite veneered multi-unit FPD, fiber-reinforced composite resinbonded FPD, conventional removable partial denture (RPD), RPD with extracoronal attachment and combination of fixed and removable partial dentures. ${ }^{7-10}$ Herein, prosthodontist should make a decision whether to use fixed (conventional or implant supported) or removable partial denture. ${ }^{7}$

RPDs are particularly indicated in patients with tissue deficiency, several fistulae, soft palate dysfunction, or uncoordinated nasopharyngeal sphincter action that can cause hypernasal speech. ${ }^{7}$ Moreover, in the circumstances of vertical bone loss at edentulous anterior region, RPDs can hinder severe hygiene problems and deficiencies of labial support contrary to FPDs ${ }^{11}$ and can provide good esthetics. ${ }^{4}$ In addition it is possible to ensure permanent retention of maxillary arc while hindering arc collapse with RPDs. ${ }^{12}$ Despite these advantages, patient satisfaction with RPDs significantly reduces with age. ${ }^{13}$ The common objection is that its removable structure accentuates its artificial character. ${ }^{4}$ Alternatively osseointegrated implants after secondary bone grafting have provided an invasive treatment approach for cleft lip and palate patients. ${ }^{14-16}$ The use of endosseous implants for CLP patients with grafted alveolar clefts have lots of advantages, especially preservation of tooth structure in healthy adjacent teeth ${ }^{17}$ and the prevention of resorption of grafted bone. ${ }^{18}$ Secondary bone grafting at the early stage of the mixed dentition posses many benefits, however there is a conflict on timing of bone grafting. ${ }^{19}$

Despite the advantages of surgical intervention in CLPs, conventional prosthetic rehabilitation may be preferred especially in young patients whom bone grafting followed 
by endosseous implants is not a treatment option. In the presented case series, 7 patients were presented describing the conventional prosthetic management of patients showing different patterns of cleft lip and palate.

\section{CASE SERIES}

Seven patients, who were treated orthodontically and received a number of different lip and palate surgeries, were referred to Baskent University, Faculty of Dentistry, Department of Prosthodontics. Of these 7 patients, 5 were female (aged 17-21 years) and 2 were male (aged 15-29 years) with a mean age of 20 years. Three patients had BCLP and 4 patients had UCLP. Two of the BCLP patients and one of the UCLP patients had triangular alveolar defect extending to the palate in which food impacted during mastication, and caused speech alteration. Rest of the patients had only alveolar defects. All of the patients had missing maxillary lateral teeth, and 3 of the patients had additional missing teeth; bilaterally maxillary central and opposite lateral incisors. Only 2 patients had morphologically abnormal teeth; as maxillary left central incisors. Maxillary arch was orthodontically expanded in 5 of the 7 patients.

Prosthetic rehabilitation was commenced with an oral hygiene protocol which is a prior condition for this form of treatment. Two types of prosthesis, based on conservative and economically feasible principals were designed. Five patients whose maxillary arch was orthodontically expanded were treated with a combination of fixed and removable partial dentures. The other 2 patients, who did not have maxillary collapse prior to orthodontic treatment, were treated either with three or four unit metal-ceramic FPDs.

For FPDs, an abutment tooth on each end of the edentulous space was used to restore missing maxillary lateral. Metalceramic FPDs were constructed with routine clinical and laboratory process. (Figs 1A to D) For combination of fixed and RPDs, the numbers of prepared abutment teeth were determined according to biomechanical considerations. ${ }^{20}$ Bar attachments on a bar spanning an edentulous area joining teeth were used. Multi-unit metal-ceramic FPDs with bar attachment were constructed. Prosthodontic treatment was completed with combined bar and clasp retention RPDs. Aker's clasp or double Aker's clasps were used on posterior teeth. Special care was shown to cover the palatal defect area with the RPD framework for enhancing speech (Figs 2A to G). All patients were followed for up to minimum of 1 year and maximum of 2 years.

\section{DISCUSSION}

Patients with congenital and craniofacial defect are unique, and oral problems must be evaluated individually to the
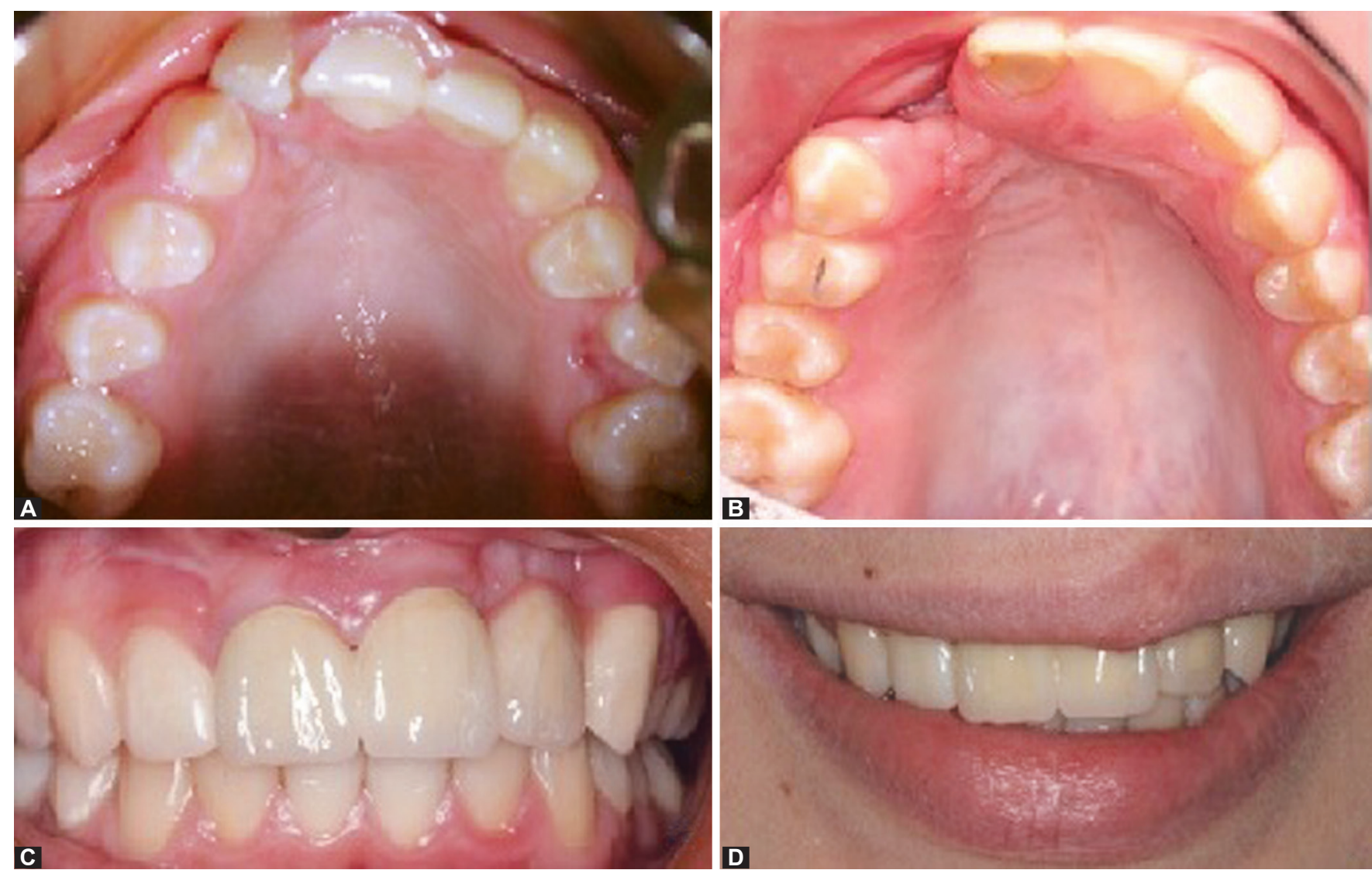

Figs 1A to D: Patient rehabilitated with FPDs. (A) Preorthodontic view, (B) preprosthetic palatal view,

(C) frontal view of FPDs, (D) esthetic view of final restoration 

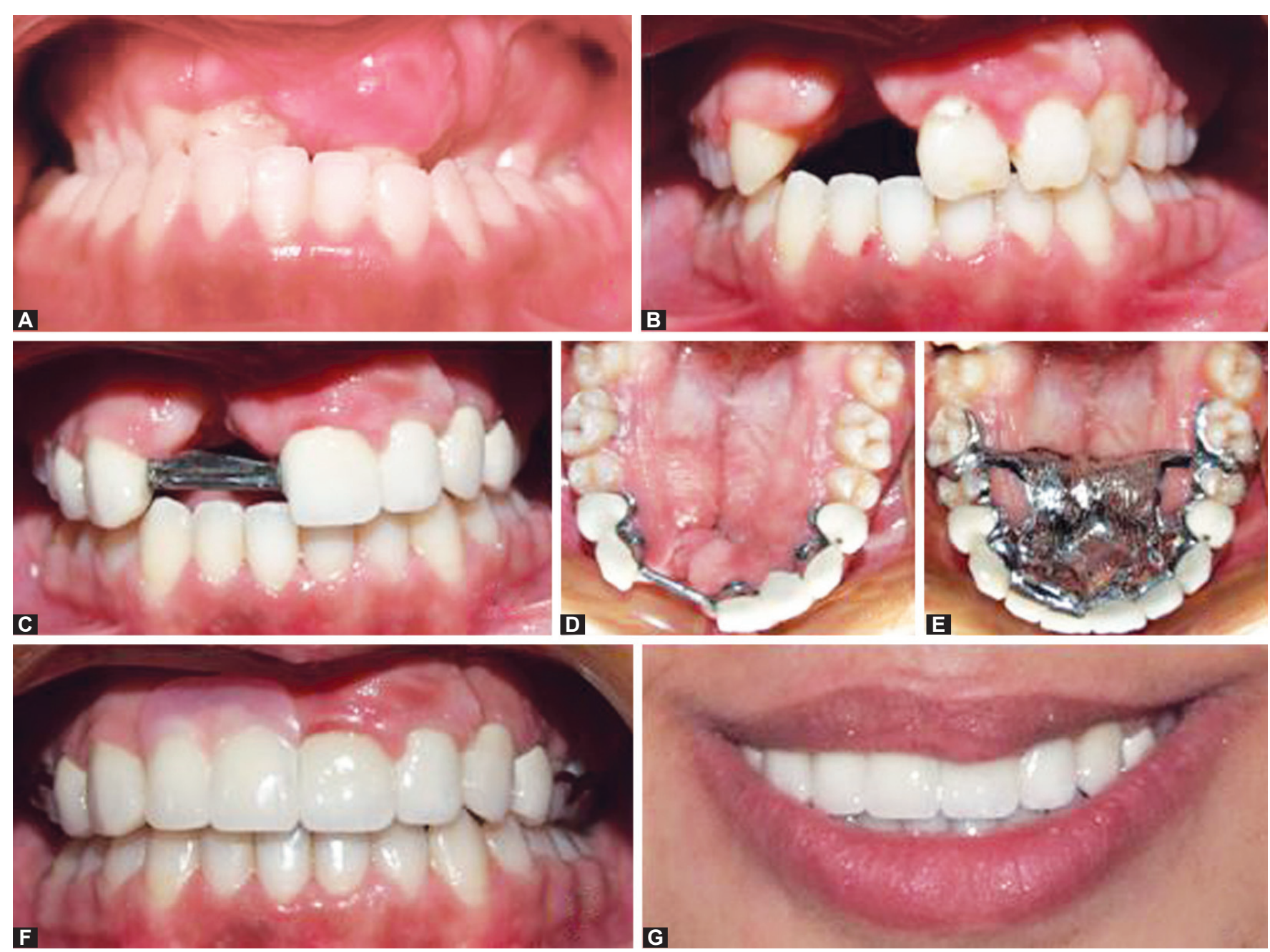

Figs 2A to G: Patient rehabilitated with RPD and FPDs. (A) Preorthodontic view, (B) postorthodontic view, (C) frontal view of bar attachment between FPDs, (D) palatal view of FPDs and palatal defect side, (E) coverage of palatal defect with RPD, (F) intraoral view with the RPD in place, $(\mathrm{G})$ esthetic view of final restoration

most ideal treatments. ${ }^{6}$ In the presented case series, all of the patients were treated with conventional fixed or removable prosthetic dentures with precision attachments. When making a decision whether to use fixed or removable partial dentures; defect form, speech and swallowing difficulties, dental abnormalities, cosmetic deformities, maxillary collapse ${ }^{7}$ age,${ }^{13}$ and financial status are the factors that form the prosthetic treatment plan.

In this case series, 3 of the patients showed a defect located in the palate, alveolar ridge and labial vestibule. Although the defects do not cause serious feeding problems, speech had been clearly affected in these patients. It was not easy to understand the patients' speech when the RPD was taken out. It is emphasized that, palatal cover of the framework assists speech therapy for correction of compensatory articulations and contributes to normal speech production. $^{4,7}$

Prosthodontic rehabilitation of missing maxillary anterior teeth requires special consideration to restore esthetics. The importance of oral health and hygiene cannot be ignored.
Besides, following surgical repair of the lip and palate, a defect in the alveolar ridge at the edentulous area adjacent to the abutment teeth may remain. ${ }^{21}$ Destruction type will play an important role in selecting the pontic design. Ridge deformities have been classified into three categories. Class I; loss of faciolingual ridge width with normal apicocoronal height, class II; loss of ridge height with normal width, and class III, loss of both ridge width and height. ${ }^{22}$ One of the alternative solutions used in the restoration of large ridge defect, particularly in the anterior segment, is the Andrews bridge system. ${ }^{23}$ It utilizes fixed retainers that are connected by a rectangular bar which fallows the curve of the ridge under it. Although the removable flange may cause plaque accumulation if not cleaned appropriately, it still may be the best way of handling large ridge defects ${ }^{23}$ as far as esthetics, phonetics and function is concerned. In the present study, patients strongly requested to restore their missing teeth with fixed restorations. Unfortunately, clinic examination showed that making a FPD including anterior missing incisors may have caused food impaction 
on the class II and III buccal defect side. ${ }^{22}$ Additionally the amount of periodontal destruction is more pronounced in the cleft lip and palate patients compared with noncleft patients. ${ }^{24}$ Bacterial plaque accumulation is enhanced due to the irregularly positioned teeth or displaced teeth, difficulty in closing lips, mouth breathing, and inadequate personal oral hygiene care. ${ }^{25}$ Therefore, the patient was rehabilitated with metal ceramic FPD with a bar attachment passing throughout the defect side.

When a missing tooth is to be replaced, a conventional $\mathrm{FPD}^{20}$ or implant supported $\mathrm{FPD}^{17}$ is preferred by the majority of the patients. However, there is an important prerequisite for FPDs, that there should be no gross soft tissue deficiency in the alveolar ridge. If there is, it may be possible to augment the ridge with grafts. ${ }^{20}$ Particularly in the CLP patients, secondary bone grafting at the early stage of the mixed dentition offers several benefits, such as bone support for unerupted teeth adjacent to the cleft, closure of oronasal fistulae, support and elevation of alar base on the cleft side, construction of a continuous arch form and alveolar ridge and stabilization of premaxilla in patients with a bilateral cleft. However, there are strong controversies concerning alveolar bone grafting related to the timing of the grafting, sequencing of orthodontic treatment to correct transverse discrepancy and type of bone for the graft. Ideally the permanent canine root must be formed half to two thirds at the time of grafting, which is generally between the ages 8 and 11. Occasionally the graft may be placed at an earlier age to improve the periodontal support of a lateral incisor. It is important to note that once teeth have erupted into the cleft side, bone grafting will not improve their periodontal support or the height of the alveolar bone crest. ${ }^{19}$ All the patients presented in this case series were those who did not want to take the potential failure risk of bone grafting related to advanced age, and additionally those who resisted secondary alveolar bone grafting due to poor economic conditions unlikely to cover the additional graft.

It is well known that the scar tissue and deficiency of palatal and alveolar bone structure cause relapse of orthodontic treatment in CLP patients. No matter how ideal the occlusion obtained or the teeth positioned, relapse is inevitable even after 10 years use of retention appliances following orthodontic treatment. It was also shown that late secondary bone grafting which was expected to increase stability of the treatment could not stabilize the maxillary transverse dimension obtained by expansion. Therefore, permanent retention with an appropriate type of prosthetic restoration is strongly recommended for these patient. ${ }^{12,26,27}$ It requires either a removable palatal prosthesis or a fixed bridge spanning the cleft. ${ }^{12}$ According to Kantorowitcz, ${ }^{28}$ FPDs should be extended as far as second premolar or the first molar to ensure stability. In this case series maxillary arch was orthodontically expanded in 5 of the 7 patients. Fixed partial denture combined with removable partial dentures attached on the crowns stabilized the premaxilla, thereby preventing relapse of the palatal expansion. ${ }^{7}$ The patients were advised to wear the RPD continuously throughout the day and night in order to maintain arc width because maxillary dental arc segments may have contracted after surgical repair of the palate. ${ }^{12}$

\section{CONCLUSION}

Despite recent advances in bone grafting and dental implants for CLPs, conventional prosthodontic rehabilitation remains to be an important aspect in treatment of these patients. The presented cases had been followed up for up to 2 years and none of them revealed bone loss around abutment teeth or any complications related to maxillary relapse. Masticatory and speech functions had markedly improved in all patients following treatment as well their psychological state. Longer follow-up periods must be made for further evaluation of these patients in terms of prosthodontic complications which may come up in the future.

\section{REFERENCES}

1. Coupland MA, Coupland AI. Seasonality, incidence, and sex distribution of cleft lip and palate births in trent region, 19731982. Cleft Palate J 1988;25:33-37.

2. Regezzi JA, Sciubba JJ, Jordan RCK, editors. Oral Pathology, Clinical Pathologic Correlations. St. Louise, Missouri, Elsevier, Saunders: 2003;362-363.

3. Paranaiba LM, Coletta RD, Swerts MS, Quintino RP, de Barros LM, Martelli-Junior H. Prevalence of dental anomalies in patients with nonsyndromic cleft lip and/or palate in a brazilian population. Cleft Palate Craniofac J 2013;50:400-405.

4. Reisberg DJ. Dental and prosthodontic care for patients with cleft or craniofacial conditions. Cleft Palate Craniofac J 2000; 37:534-537.

5. Moore D, McCord JF. Prosthetic dentistry and the unilateral cleft lip and palate patient. The last 30 years. A review of the prosthodontic literature in respect of treatment options. Eur J Prosthodont Restor Dent 2004;12:70-74.

6. Hickey AJ, Salter M. Prosthodontic and psychological factors in treating patients with congenital and craniofacial defects. J Prosthet Dent 2006;95:392-396.

7. Hochman N, Yaffe A, Brin I, Zilberman Y, Ehrlich J. Functional and esthetic rehabilitation of an adolescent cleft lip and palate patient. Quintessence Int 1991;22:401-404.

8. Watanabe I, Kurtz KS, Watanabe E, Yamada M, Yoshida N, Miller AW. Multi-unit fixed partial denture for a bilateral cleft palate patient: a clinical report. J Oral Rehabil 2005;32:620-622.

9. Mese A, Ozdemir E. Removable partial denture in a cleft lip and palate patient: a case report. J Korean Med Sci 2008;23: 924-927.

10. Ayna E, Basaran EG, Beydemir K. Prosthodontic rehabilitation alternative of patients with cleft lip and palate (CLP): two case reports. Int J Dent 2010;DOI:10.1155/2009/515790. 
11. Preiskel HW. Precision attachments in prosthodontics: the applications of intracoronal and extracoronal attachents. London: Quintessence Publishing Co., Inc. 1984 p.151-154.

12. Berkowitz S. Cleft lip and palate: diagnosis and management. Berlin: Springer; 2006:132.

13. Koyama S, Sasaki K, Yokoyama M, Sasaki T, Hanawa S. Evaluation of factors affecting the continuing use and patient satisfaction with removable partial dentures over 5 years. J Prosthodont Res 2010;54:97-101.

14. Kawakami SY, Horiuchi M, Moriyama S, Oral K. Rehabilitaion of an orthodontic patient with cleft lip and palate and hypodntia using secondary bone grafting, osseointegrated implants, and prosthetic treatment. Cleft Palate Craniofac J 2003;41:279-284.

15. Fukuda M, Takahashi T, Iino M. Dentoalveolar reconstruction of a missing premaxilla using bone graft and endosteal implants. J Oral Rehabil 2003;30:87-90.

16. Verdi FJ Jr, SLanzi GL, Cohen SR, Powell R. Use of the Branemark implant in the cleft palate patient. Cleft Palate Craniofac J 1991;28:301-303.

17. Mish C. Dental Implant Prosthetics. St Louise, Missouri: Elsevier Mosby. 2005;4.

18. Kearns G, Perrott DH, Sharma A, Kaban LB, Vargervik K. Placement of endosseous implants in grafted alveolar clefts. Cleft Palate Craniofac J 1997;34:520-525.

19. Graber TM, Vanarsdall RL, Vig KWL, editors. Orthodontics: current principles and techniques, 4th ed. St Louis: Elsevier Mosby; 2005.

20. Shilinburg HT. Fundamentals of fixed Prosthodontics. Quintessence Publishing Co, Inc: 1997;85-103.

21. Tan AE, Henry PJ. Periodontal implications of the adolescent cleft palate patient. Aust Dent J 1985;30:8-14.

22. Seibert JS. Reconstruction of deformed, partially edentulous ridges, using full thickness onlay grafts. Part I. Technique and wound healing. Compend Contin Educ Dent 1983;4:437-453.

23. Seibert JS, Cohen DW. Periodontal considerations in preparation for fixed and removable prosthodontics. Dent Clin North Am 1987;31:529-555.
24. Bragger U, Schurch E Jr, Salvi G, von Wyttenbach T, Lang NP. Periodontal conditions in adult patients with cleft lip, alveolus, and palate. Cleft Palate Craniofac J 1992;29:179-185.

25. Bragger U, Schurch E Jr, Gusberti FA, Lang NP. Periodontal conditions in adolescents with cleft lip, alveolus and palate following treatment in a coordinated team approach. J Clin Periodontol 1985;12:494-502.

26. Ulgen M. Orthodontic treatment principles. Ankara: Ankara University Faculty of Dentistry Press, 2010;512-527.

27. Enacar A. The control of midfacial growth in cleft lip and palate: maxillary orthopedic and orthodontic treatment. In: Cleft Lip and Palate. Ankara 1999;303-326.

28. Kantarowitcz GF. Bridge prosthesis for left cleft palate patients. Br Dent J 1975;139:91-97.

\section{ABOUT THE AUTHORS}

\section{Ozlem Acar (Corresponding Author)}

Research Assistant, Department of Prosthodontics, Baskent University Ankara, Turkey, Phone: 903122151336, e-mail: zlemacr@gmail.com

\section{Burcak Kaya}

Assistant Professor, Department of Orthodontics, Baskent University Ankara, Turkey

\section{Muhammet Saka}

Research Assistant, Department of Prosthodontics, Baskent University Ankara, Turkey

\section{Bulem Yuzugullu}

Associate Professor, Department of Prosthodontics, Baskent University, Ankara, Turkey 